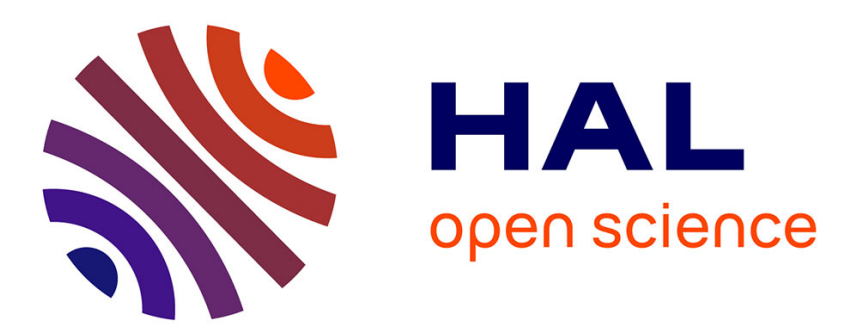

\title{
Comparing the solar minima of cycles $22 / 23$ and 23/24: The view from LASCO white light coronal images
}

\author{
P. Lamy, T. Barlyaeva, A. Llebaria, O. Floyd
}

\section{To cite this version:}

P. Lamy, T. Barlyaeva, A. Llebaria, O. Floyd. Comparing the solar minima of cycles 22/23 and 23/24: The view from LASCO white light coronal images. Journal of Geophysical Research Space Physics, 2014, 119 (1), pp.47-58. 10.1002/2013JA019468 . hal-01441000

\author{
HAL Id: hal-01441000 \\ https://hal.science/hal-01441000
}

Submitted on 12 Nov 2021

HAL is a multi-disciplinary open access archive for the deposit and dissemination of scientific research documents, whether they are published or not. The documents may come from teaching and research institutions in France or abroad, or from public or private research centers.
L'archive ouverte pluridisciplinaire HAL, est destinée au dépôt et à la diffusion de documents scientifiques de niveau recherche, publiés ou non, émanant des établissements d'enseignement et de recherche français ou étrangers, des laboratoires publics ou privés. 


\section{Journal of Geophysical Research: Space Physics}

\section{RESEARCH ARTICLE}

10.1002/2013JA019468

\section{Special Section:}

The Causes and Consequences of the Extended Solar Minimum between Solar Cycles 23 and 24

Key Points:

- The global radiance of the $K$ corona follows the activity pattern of the Sun - It is $24 \%$ fainter at the minimum of cycle 23/24 compared to cycle 22/23 - However, the two hemispheres experienced different reductions

\section{Correspondence to:}

P. Lamy,

philippe.lamy@oamp.fr

\section{Citation:}

Lamy, P., T. Barlyaeva, A. Llebaria, and O. Floyd (2014), Comparing the solar minima of cycles $22 / 23$ and $23 / 24$ : The view from LASCO white light coronal images, J. Geophys. Res. Space Physics, 119, 47-58, doi:10.1002/2013JA019468.

Received 23 SEPT 2013

Accepted 15 DEC 2013

Accepted article online 19 DEC 2013

Published online 21 JAN 2014

\section{Comparing the solar minima of cycles $22 / 23$ and $23 / 24$ : The view from LASCO white light coronal images}

\author{
P. Lamy' ${ }^{1}$, T. Barlyaeva' ${ }^{\text {, A. Llebaria }}{ }^{\text {, , and O. Floyd' }}$ \\ 'Laboratoire d'Astrophysique de Marseille, UMR 7326, CNRS and Aix-Marseille Université, Marseille, France
}

Abstract The Large Angle and Spectrometric Coronagraph LASCO-C2 aboard SOHO has now completed 17 years (1996-2012) of quasi-continuous white-light imaging of the corona from 2.2 to 6.5 solar radii, thus allowing an unprecedented view of its evolution over a solar cycle and a half including the minima of solar cycles $22 / 23$ and $23 / 24$. The corrected and calibrated polarization sequences produce images of the radiance $(B)$, the polarized radiance $(p B)$, and the electron density $N_{e}$ of the $K$ corona, and, in turn, of their synoptic maps. Their temporal variations are quantified by integration first globally, then in the north and south hemispheres, and finally, in sectors of $30^{\circ}$ latitudinal extent centered along the equatorial and polar directions. The global radiance of the $K$ corona follows well the solar activity as described by the sunspot number and the radio flux and was $24 \%$ fainter during the minimum of solar cycle $23 / 24$ than during that of cycle $22 / 23$. However, the two hemispheres experienced different reductions, $17 \%$ for the north one and $29 \%$ for the south one. The equatorial sector suffered a drastic reduction of $44 \%$, in remarkable agreement with the in situ measurements of Wind and ACE at $1 \mathrm{AU}$, whereas the north and south polar sectors did not experience much variation. Cycle 23 is estimated to have lasted 12 years and 3 months. Maximum conditions have been reached in the northern region, whereas the southern region is still lagging. Finally, the rate of coronal mass ejections follows well the solar activity.

\section{Introduction}

The question of the temporal variation of the solar white light corona and its connection to the solar cycle has been considered since the middle of the 20th century, see Fisher and Sime [1984] for an historical review. The change in the spatial structure of the corona was perceived as eclipse observations accumulated. In a broad stroke, the ellipticity of the isophotes of the coronal brightness or radiance, quantified by the Ludendorff number, was found to reach its maximum near solar minimum and to progressively decrease tending to become circular near the time of solar maximum. Following progress made in the photometric analysis of the corona, the temporal variation of its total brightness was further investigated leading to rather conflicting results ranging from no variation to an increase of various amplitudes as the activity cycle progresses from solar minimum to maximum.

This question has been primarily investigated on the basis of eclipse observations which however present several shortcomings. First, an obvious limitation is time sampling, typically every few years. Second, diverse instruments and calibration techniques have been implemented resulting in a lack of homogeneity of the quality of the measurements not to mention the role of varying sky backgrounds. Third, they have been performed either on the total $K+F$ corona or on the $K$ corona alone after proper separation and in different regions of the corona, both aspects possibly responsible for distortions or biases in the results. $K$ coronameters recording the integrated polarized brightness $(p B)$ of the corona have alleviated these difficulties to a large extent. Analyzing an 18 year long record from the Mauna Loa, Hawaii instruments, Fisher and Sime [1984] determined an increase of a factor of 2 (measured at reference heights of 1.3 and $1.5 R_{\odot}$ ) between the minimum and maximum of the activity cycle. No particular attention was given to the comparison of successive solar minima.

In principle, space coronagraphy is the almost perfect solution to the question of the variability of the corona, alleviating all shortcomings mentioned above, but none of the instruments on past long missions, Skylab, Solwind, and Solar Maximum Mission, had the proper photometric calibration to perform quantitative studies. The Large Angle Spectrometric Coronagraph (LASCO) [Brueckner et al., 1995] with its superior sensitivity, excellent photometric performances, and record longevity has opened a new era in quantitative 
analysis of the white light corona over a long period of time. In practice, the field of view of LASCO has an inner limit of $2.2 \mathrm{R}_{\odot}$ thus excluding the brightest part of the $K$ corona, but it turns out from previous analysis and as will be obvious later in the present study that this does not present a problem for the question of the temporal variation of the solar white light corona and its connection to the solar cycle. Llebaria et al. [1999] and Lamy et al. [2002] have already considered this question but on a limited time frame compared to the 17 years of data (1996 to 2012 inclusive) now available. Nevertheless, they have shown that the integrated radiance of the $K$ corona and its global electron content follow very well the solar cycle pattern given for instance by the international sunspot number (SSN).

In the present article, we analyze the temporal variations of the radiance of the $K$ corona and of the electron density based on observations obtained with the LASCO-C2 coronagraph and concentrate on the comparison between the minima of solar cycles $22 / 23$ and $23 / 24$, the latter being often referred as an anomalous minimum. The complete temporal analysis of the full data set covering the first 17 years of LASCO will be presented in a separate publication (T. Barlyaeva et al., manuscript in preparation, 2014). This article is organized as follows. In section 2, we describe the LASCO-C2 data set and its processing leading to the generation of calibrated maps of the radiance and of the polarized radiance of the $K$ corona, of maps of the electron density, and of their synoptic maps. In section 3 , we analyze the temporal variation of the integrated radiance, globally and in selected sectors and perform the comparison between the two minima. In section 4, we discuss the differences of the morphology of the streamer belt during the two minima. Section 5 is devoted to the temporal variation of the rate of coronal mass ejections. In section 6 we discuss our results and put them in perspective with other studies and findings. Finally, we summarize them and conclude in section 7.

\section{The LASCO-C2 Data Set and Its Processing}

$\mathrm{C} 2$ is one of the two externally occulted coronagraphs of LASCO which has been in nearly continuous operation since January 1996. With its field of view extending from 2.2 to $6.5 \mathrm{R}_{\odot}$, it records the brightest part of the corona accessible to LASCO, and its images are therefore well suited to investigate its temporal variation. The operation of LASCO observations has experienced minor interruptions since 1996 for various instrumental and spacecraft reasons except from 25 June to 22 October 1998 when the SOHO spacecraft was mistakenly pointed in the wrong direction. However, after recovery, normal operations did not resume until March 1999. The routine observational program is basically composed of unpolarized, "white light" images of $1024 \times 1024$ pixels every 20 min and a daily polarization sequence all taken with an orange filter (band pass of 540-640 nm); in addition, there has been specific campaigns usually coordinated with other SOHO instruments.

The present analysis makes use of these polarization sequences since their temporal cadence is sufficient for our purpose and since they allow to extract the $K$ corona following a classical procedure to be described below. A polarization sequence is composed of three linear polarized images of the corona obtained with three polarizers oriented at $60^{\circ}, 0^{\circ}$, and $-60^{\circ}$ and an unpolarized image, all taken with the orange filter in the binned format of $512 \times 512$ pixels.

All LASCO images, either polarized or unpolarized, undergo a standard preprocessing which corrects for instrumental effects. The in-flight performances of $\mathrm{C} 2$ are continuously monitored so as to update these corrections as well as the absolute calibration. The present analysis further benefits from recent improvements introduced in the procedures. The preprocessing performs the following tasks.

1. Bias correction. The bias level of the CCD detector evolves with time; it is continuously monitored using specific blind zones and systematically subtracted from the images.

2. Exposure time equalization. Small random errors in the exposure times are corrected using a method developed by Llebaria and Thernisien [2001] in which relative and absolute correction factors are determined.

3. Missing block correction. Telemetry losses result in blocks of $32 \times 32$ pixels sometimes missing in the images. Different solutions are implemented to restore the missing signal depending upon the location of these blocks [Pagot et al., 2013].

4. Cosmic rays correction. The impacts of cosmic rays (and stars as well) are eliminated from the images using the procedure of opening by morphological reconstruction developed by Pagot et al. [2013]. 


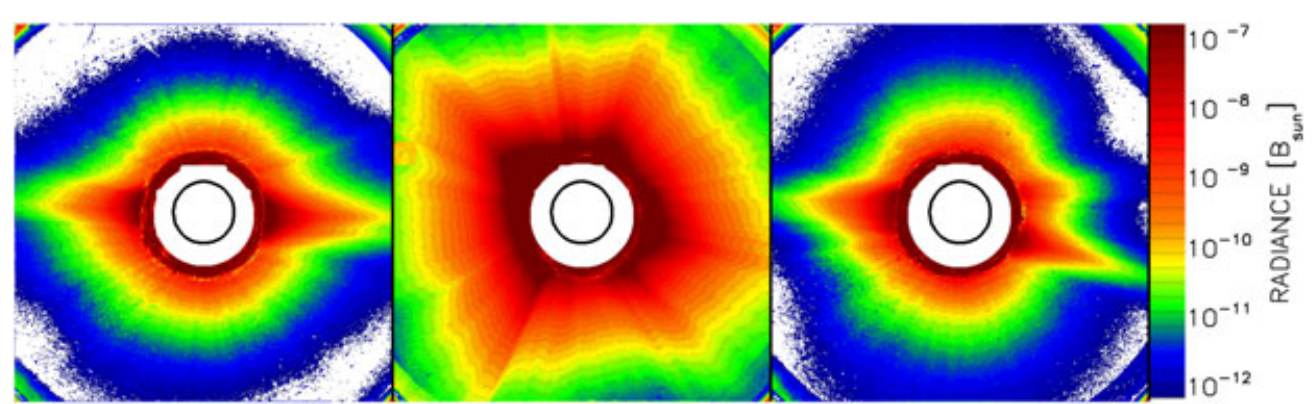

Figure 1. Three images of the $K$ corona obtained with the LASCO-C2 coronagraph (left) on 16 June 1997 during the minimum of solar cycle 22/23, (middle) on 1 January 2000 during the maximum of cycle 23, and (right) on 21 December 2008 during the minimum of solar cycle $23 / 24$. The radiance of the corona is in unit of the mean radiance of the solar disk.

The polarized images further undergo the following processes.

1. Correction for the global transmission of the polarizers.

2. Correction for the spatial variation of the transmission of the polarizers [Llebaria and Lamy, 2008].

3. Correction for the circular polarization introduced by the two folding mirrors.

4. Polarimetric analysis based on the Mueller procedure. It is applied to each triplet of polarized images and returns images of the total radiance, the polarization, and the angle of polarization.

5. Vignetting correction. This instrumental effect is removed from the radiance images using a geometric model of the two-dimensional vignetting function of C2 [Llebaria et al., 2004].

6. Absolute calibration of the total radiance derived from the photometric measurements of thousand of observations of stars present in the C2 field of view [Llebaria et al., 2006; Gardes et al., 2013].

At this stage, the images represent the sum of the radiances of the $K$ and $F$ coronae and of the instrumental stray light. The separation of the $K$ component is performed on the basis of the classical assumptions that the $F$ corona and the stray light are unpolarized based upon the fact that both mostly result from diffraction effects. This is indeed a valid assumption for the $F$ corona inside $6.5 \mathrm{R}_{\odot}$. A polarized component of the stray light would add a constant offset altering the gradient of the radial profiles of the polarized radiance $p B$ in the outer region of the corona. This is not observed as illustrated in Lamy et al. [1997, Figure 2] which displays such a profile along the direction of the North Pole for 4 February 1996. In addition, this figure shows that the signal-to-noise ratio is excellent up to $6.2 \mathrm{R}_{\odot}$. Using the above assumptions, the polarized radiance $p B$ is equal to $p_{k} B_{k}$. At this point, we use a model of $p_{K}$ calculated for a standard spherical corona [Allen, 1973], and this is justified by the robust "asymptotic" behavior of $p_{K}$ beyond $2.2 \mathrm{R}_{\odot}$. The radiance maps of the $K$ corona can then be derived, and three examples are given in Figure 1.

The electron density $N_{e}$ is computed by inversion of the $p B$ images using a two-step numerical technique which assumes local spherical symmetry for each radial distribution of electron density. In the first step, the density is approximated by a power law of the heliocentric distance and then converted to polarized radiance using the equations of van de Hulst [1950]. In the second step, a multiplicative correction factor is iteratively applied to the electron density until the calculated polarized radiance satisfactorily matches the observed one. The internal accuracy of the method is excellent as illustrated by the tests presented by Lamy et al. [1997]. The uncertainty affecting the electron density is dominated by possible systematic errors (i.e., instrumental corrections) whose discussion is beyond the scope of the present paper and from the assumption of local spherical symmetry. As we are interested here in the temporal variations, the effect of systematic errors is alleviated to a large extent.

The large-scale evolution of the corona, and particularly its streamer belt, is best tracked on synoptic maps. They are constructed from the entire sets of images by extracting profiles along circles centered on the Sun, and stacking them in rectangular arrays where the horizontal axis is time and the vertical axis is solar latitude. Our circles have radii of 2.5, 2.7, 3.0, 4.0, and 5.5 $\mathrm{R}_{\odot}$ and are sampled every $1^{\circ}$ starting at the South Pole, thus producing 360 pixels along a column. In order to avoid the problem of nonuniform sampling in time and to normalize the temporal evolution on a fixed meridian, we introduced a regular sampling with a step equal to half a mean synodic rotation, that is 13.6 days. The extraction of the circular profiles is done alternatively clockwise and counterclockwise in order to correctly track the coronal features on consecutive images. 

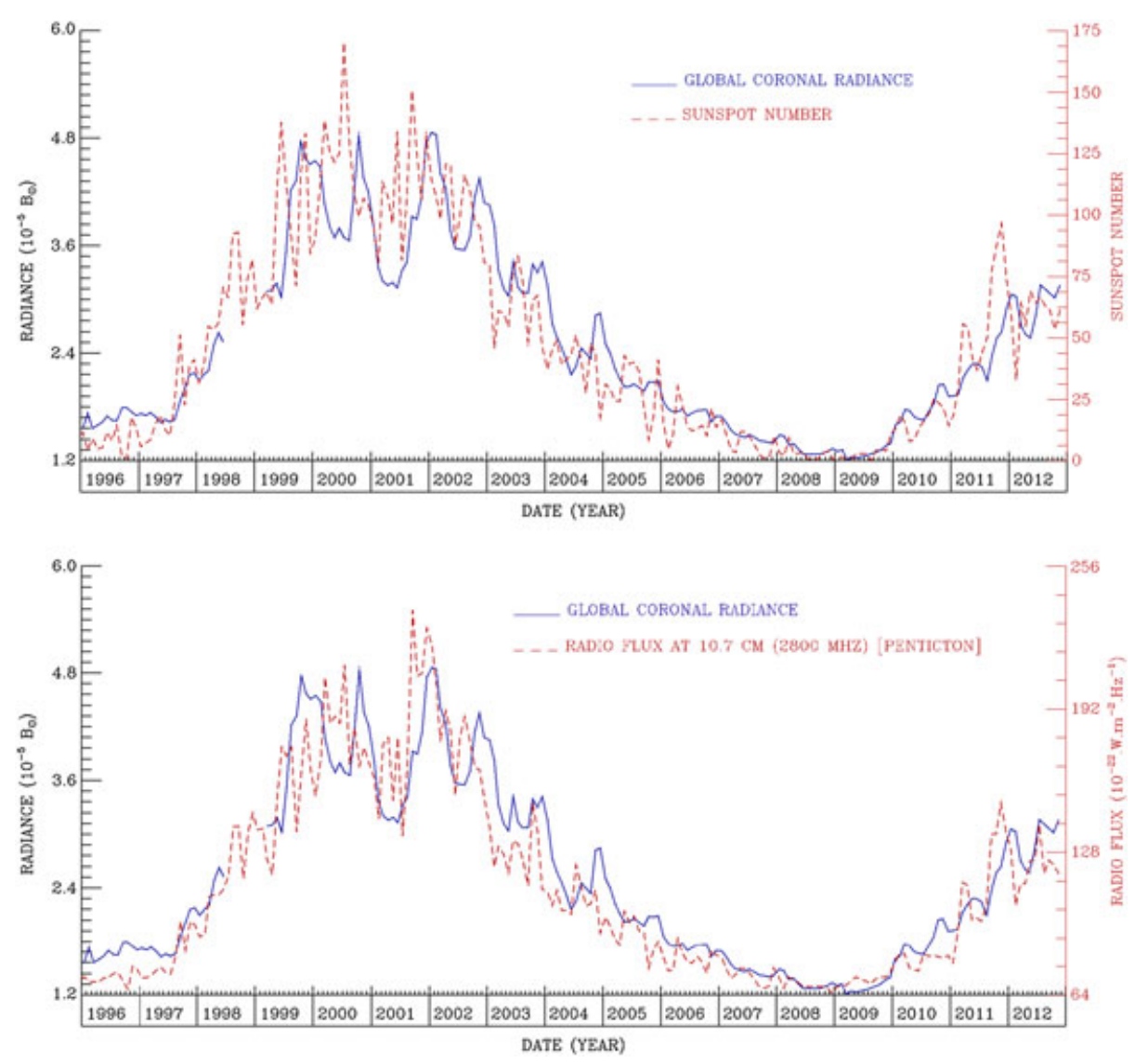

Figure 2. Temporal variation of the global radiance of the $K$ corona integrated from 2.7 to $5.5 \mathrm{R}_{\odot}$ compared with (top) the sunspot number and (bottom) the $F_{10.7}$ radio flux.

Note that these maps simultaneously display the east and west sides since the extractions are performed along full circles.

Coronal mass ejections (CMEs) are detected on synoptic maps based on their morphological appearance. The automated method is based on adaptive filtering and segmentation, followed by merging with high-level knowledge and resulted in the production of the ARTEMIS-I catalog [Boursier et al., 2009]. A new generation of high-definition maps later resulted in the present ARTEMIS-Il catalog (see detail in Floyd et al. [2013]).

\section{Temporal Variations of the Coronal Radiance}

Having generated corrected and calibrated images of the corona, we are now in position to quantify its temporal evolution. The analysis has been performed on all three quantities, radiance, polarized radiance, and electron density, but we limit the present section to the radiance for conciseness and we note that it is directly related to the electron density.

We first perform a global integration of the radiance images of the $K$ corona in an annular region extending from 2.7 to $5.5 \mathrm{R}_{\odot}$ thus slightly restricting the field of view of $C 2$ to avoid possible traces of stray light from the occulter in the inner region and dropping the outer region of low coronal signal. Because the SOHO-Sun distance varies due to the slight eccentricity of the Earth orbit (and therefore of the L2 Lagrangian point), the size of the inner and the outer radii expressed in pixels varies accordingly to precisely measure the same region of the solar corona throughout the year. We then restrict the integral to different latitude sectors in order to get a more detailed insight of the evolution. We first consider the two hemispheres north and south separately. We next introduce three latitude sectors $30^{\circ}$ wide centered on the equator (east and west sides are combined) and on the solar north and south directions. 
A

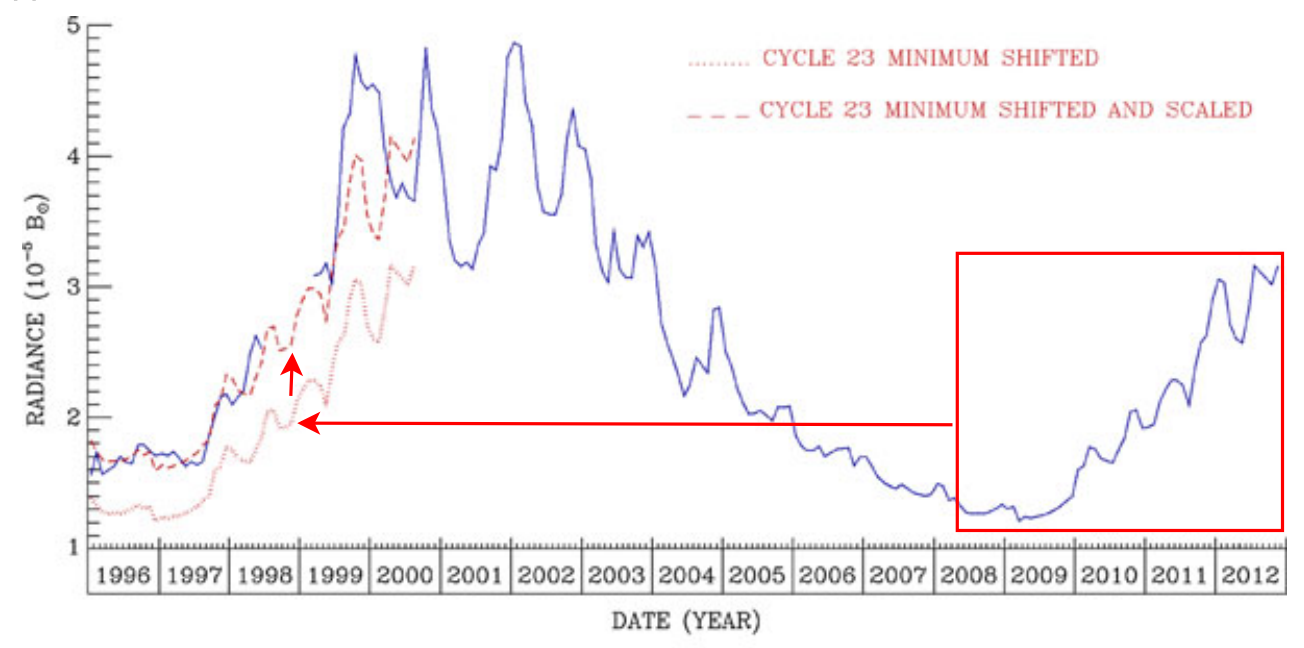

B

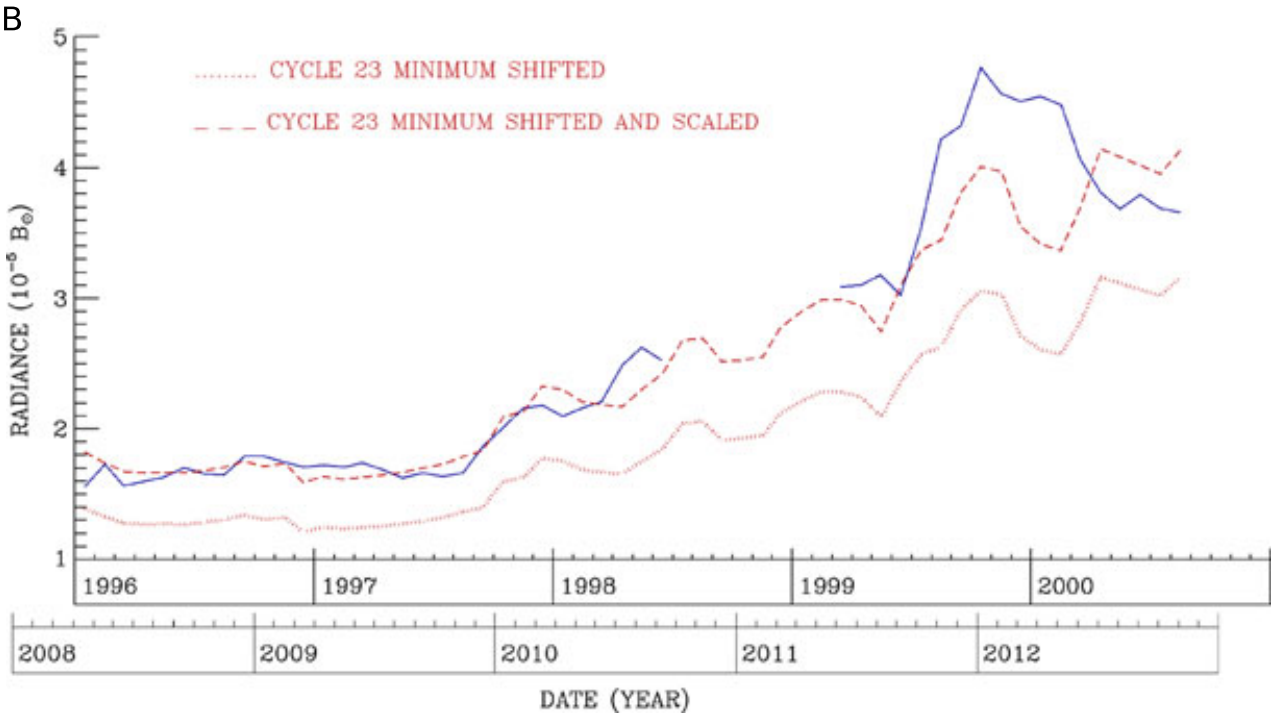

Figure 3. (a) Illustration of the process of phasing and scaling the rising branches of the global radiance of the $K$ corona of solar cycles 23 and 24. (b) An enlarged view limited to 5 years.

Figure 2 displays the monthly means of the global radiance of the $K$ corona from January 1996 to December 2012 together with the monthly mean sunspot number (SSN) and the radio flux at $10.7 \mathrm{~cm}\left(F_{10.7}\right)$. The general trend follows very well the variation of these standard proxies of solar activity thus demonstrating the close correlation of the modulation of the global radiance in phase with the solar cycle. We leave the analysis of the detailed temporal structures of the radiance curve to a forthcoming article (Barlyaeva et al., manuscript in preparation) and concentrate on the comparison of the two minima. This is best achieved by phasing the two rising branches of cycles 23 and 24 and then scaling the shifted curve to reach the best possible match. Figure 3 shows that this procedure works extremely well and yields an unambiguous time shift of 12 years and 3 months (i.e., 147 months) and a scaling factor of 0.76 . This factor was calculated by taking the ratio of the averaged radiance of the two minima over a time interval of 1.5 years during which the radiances were essentially constant (except for minor fluctuations wiped out by the averaging). This implies that the global radiance of the $K$ corona in the annular region extending from 2.7 to $5.5 \mathrm{R}_{\odot}$ was $24 \%$ fainter during the minimum of solar cycle $23 / 24$ than during the previous minimum. Since LASCO did not observe the descending branch of solar cycle 22, it is not possible to ascertain the duration of the following minimum and thus compare it with that of the minimum of cycle 23/24.

A similar procedure was applied to the two hemispheres and equatorial and polar sectors as respectively illustrated in Figures 4 and 5. Whereas the same time shift of 147 months is appropriate and was 


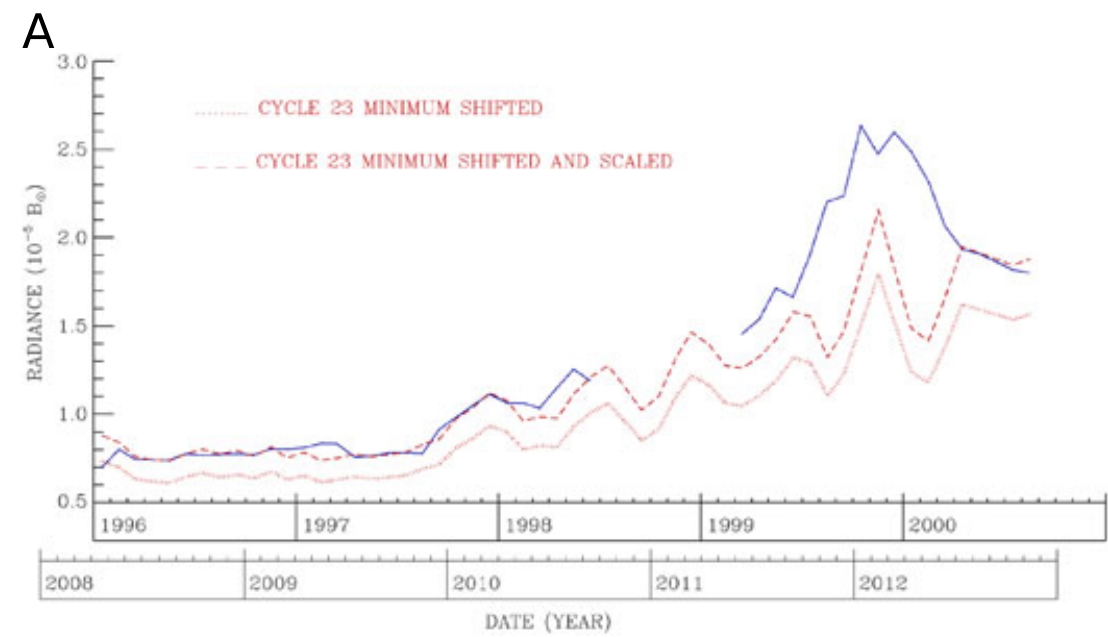

B

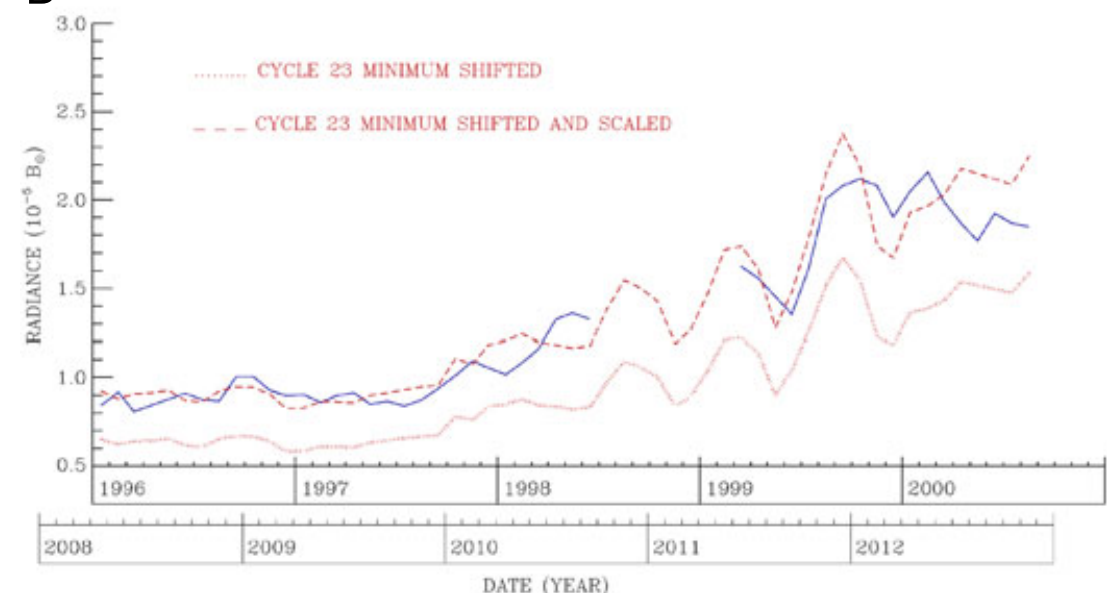

C

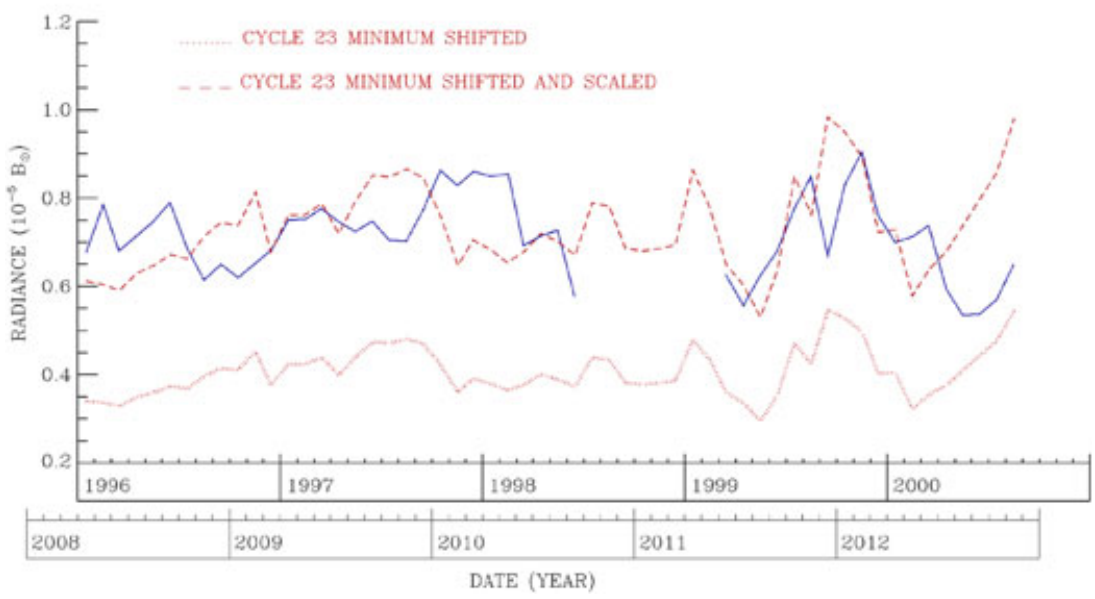

Figure 4. Results of phasing and scaling the temporal variation of the radiance of the $K$ corona during the minima of solar cycles $22 / 23$ and $23 / 24$. The radiance is integrated in different sectors: (a) north and (b) south Hemispheres, and (c) equator.

retained, the scaling factors are different as summarized in Table 1. We note a significant difference between the two hemispheres with the north one having experienced the smallest variation, which is a decrease of $17 \%$ compared to $29 \%$ for the south one. The most striking result comes from the equatorial sector which suffered a drastic reduction of $44 \%$ that is almost a factor of 2 . On the contrary, 

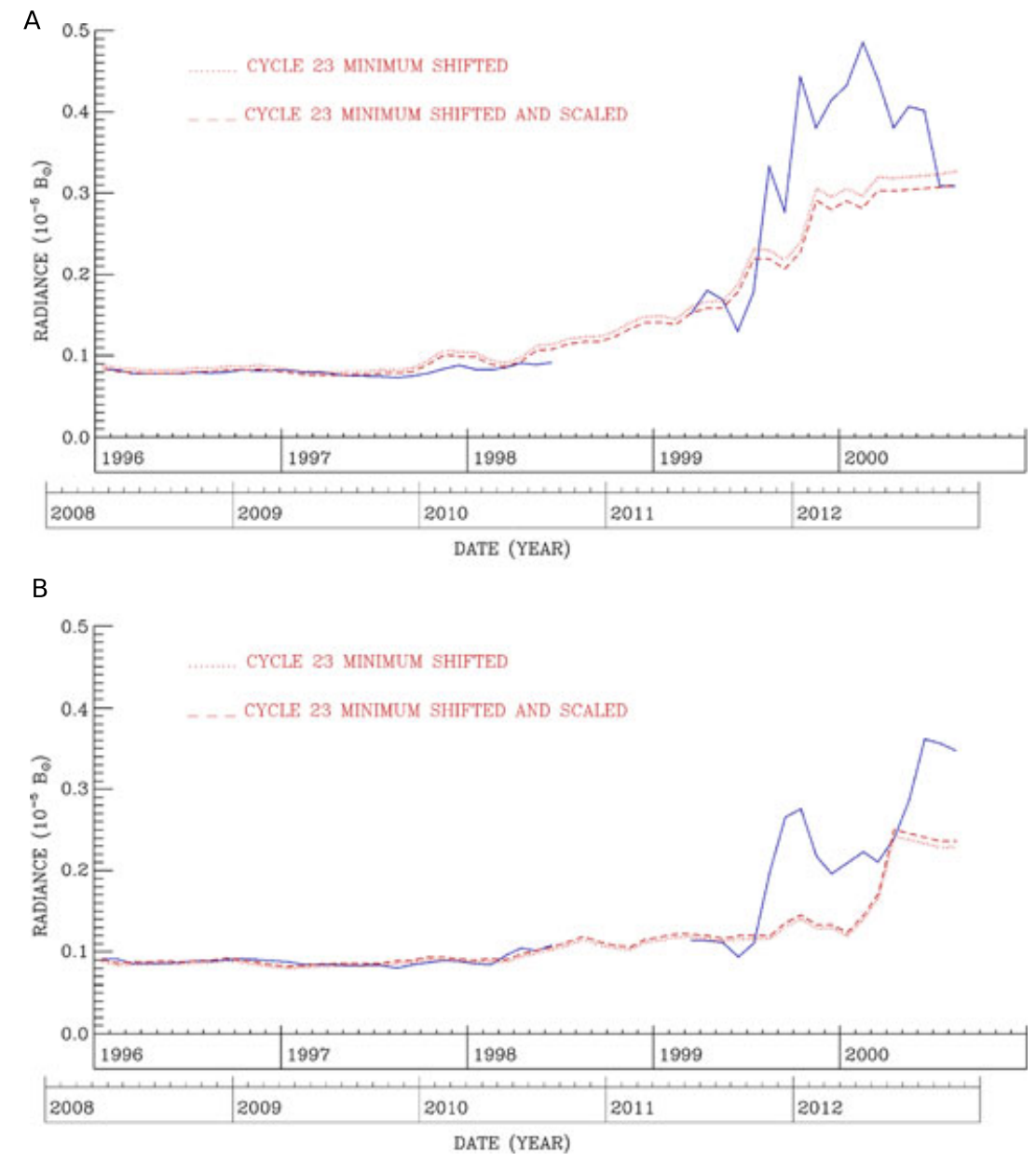

Figure 5. Results of phasing and scaling the temporal variation of the radiance of the $K$ corona during the minima of solar cycles $22 / 23$ and $23 / 24$. The radiance is integrated in different sectors: (a) north and (b) south.

the north and south polar sectors did not experience much variation, a slight increase of $5 \%$ for the north one and a modest decrease of $3 \%$ for the south one.

\section{Structure of the Corona}

An overview of the global structure of the corona during the 17 years of LASCO observations is given by the heliolatitudinal distribution of the electron density at two extreme heliocentric distances, 2.7 and $5.5 \mathrm{R}_{\odot}$ (Figure 6). The variation with the solar cycle is striking with the broadening and brightening of the streamer belt. The difference between the two minima is conspicuous and characterized by a broader and fainter equatorial belt in cycle 23/24 compared to cycle $22 / 23$. This has been shown by Manoharan [2012] to be consistent with the Ooty interplanetary scintillation data for both the solar wind speed and density turbulence. Figure 7 where the time coverage is limited to 2 years

Table 1. Scaling Factors Determined by Matching the Minima of the Radiance of the $K$ Corona During Solar Cycles 22 and 23 for the Global Corona and for Different Sectors

\begin{tabular}{lc} 
& Scaling Factor \\
\hline Global & 0.76 \\
North hemisphere & 0.83 \\
South hemisphere & 0.71 \\
Equator & 0.56 \\
North Pole & 1.05 \\
South Pole & 0.97 \\
\hline
\end{tabular}

allows a closer look at and an easier comparison of the two minima. Clearly, during the cycle 23/24 minimum, the solar corona never reached the simple "dipole" geometry often observed during solar minima. During the declining phase of cycle 22, a simple warping of the heliospheric current sheet progressively vanishing explains the decaying oscillation of the streamer belt which became essentially flat and narrow. On the contrary, during 


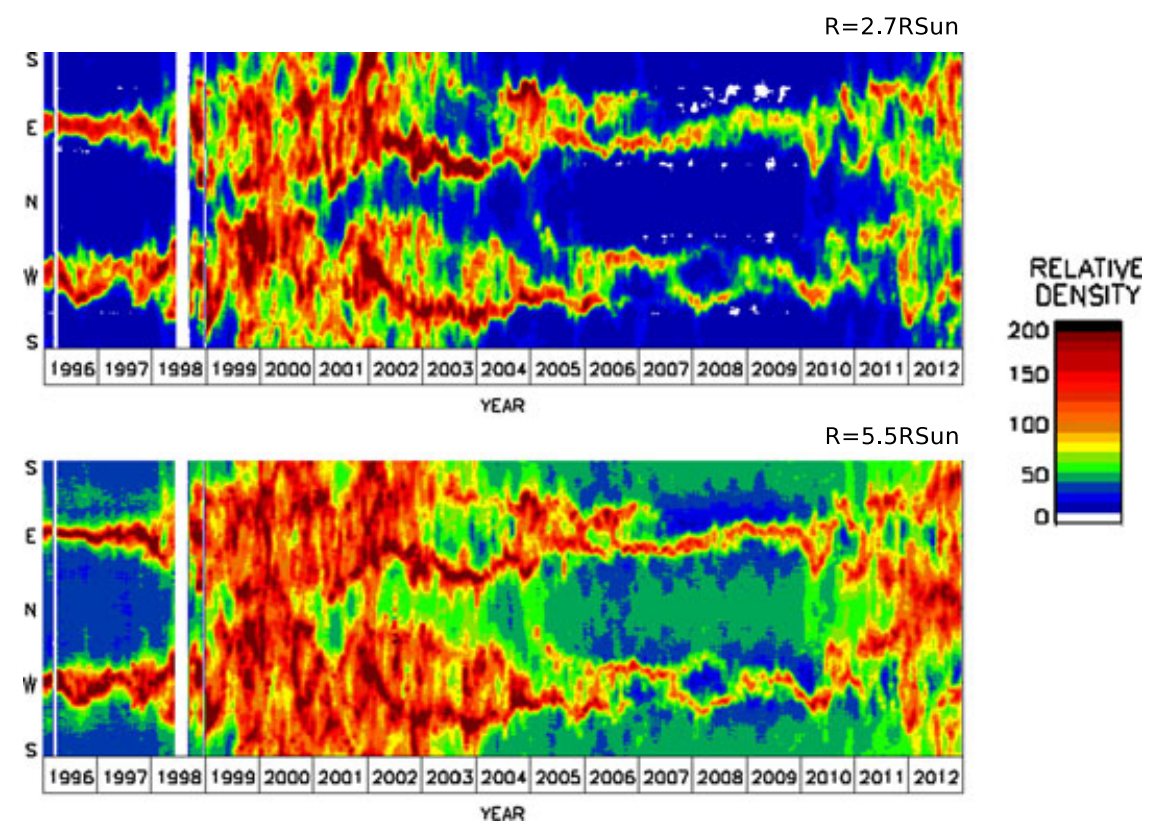

Figure 6. Heliolatitudinal distribution of the coronal electron density at two heliocentric distances, (top) $2.7 \mathrm{R}_{\odot}$ and (bottom) $5.5 \mathrm{R}_{\odot}$.

the minimum of cycle $23 / 24$, the structure of the corona and consequently of the underlying magnetic field remained relatively complex, and the equatorial belt occupied a broader latitude range (see also Figure 1). Consequently, the coronal holes never reached the very low latitudes of approximately $15^{\circ}$ to $20^{\circ}$ typical of solar minima.
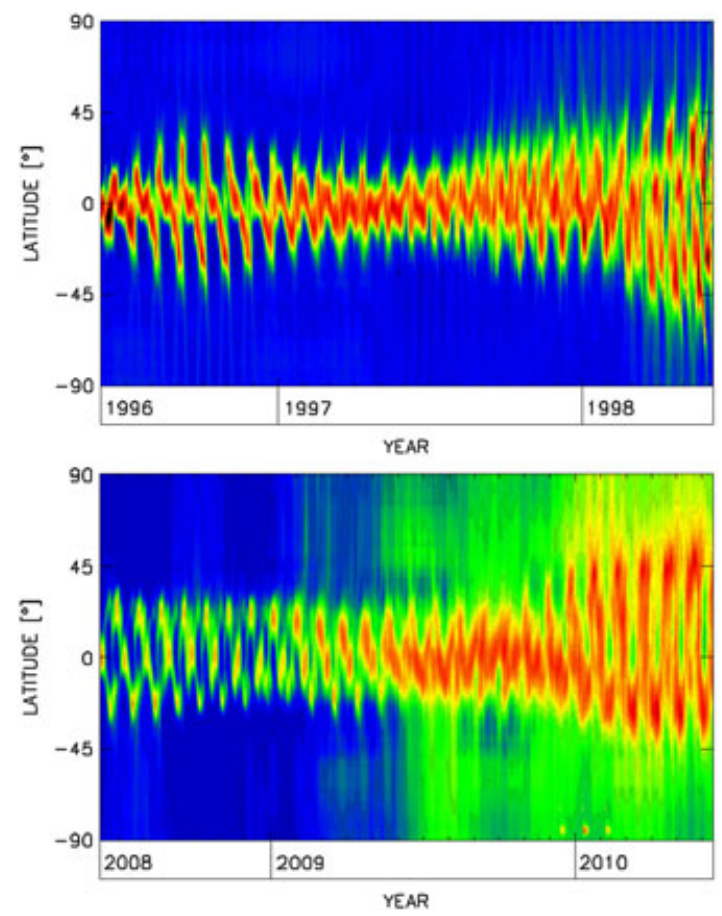

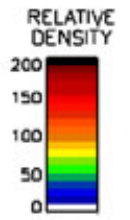

Figure 7. Enlarged extracts of Figure 6 displaying the electron density at a heliocentric distance of $2.7 \mathrm{R}_{\odot}$ extending over approximately 2 years (27 Carrington rotations) centered on the minimum of (top) solar cycle 22/23 and on that of (bottom) cycle 23/24. 

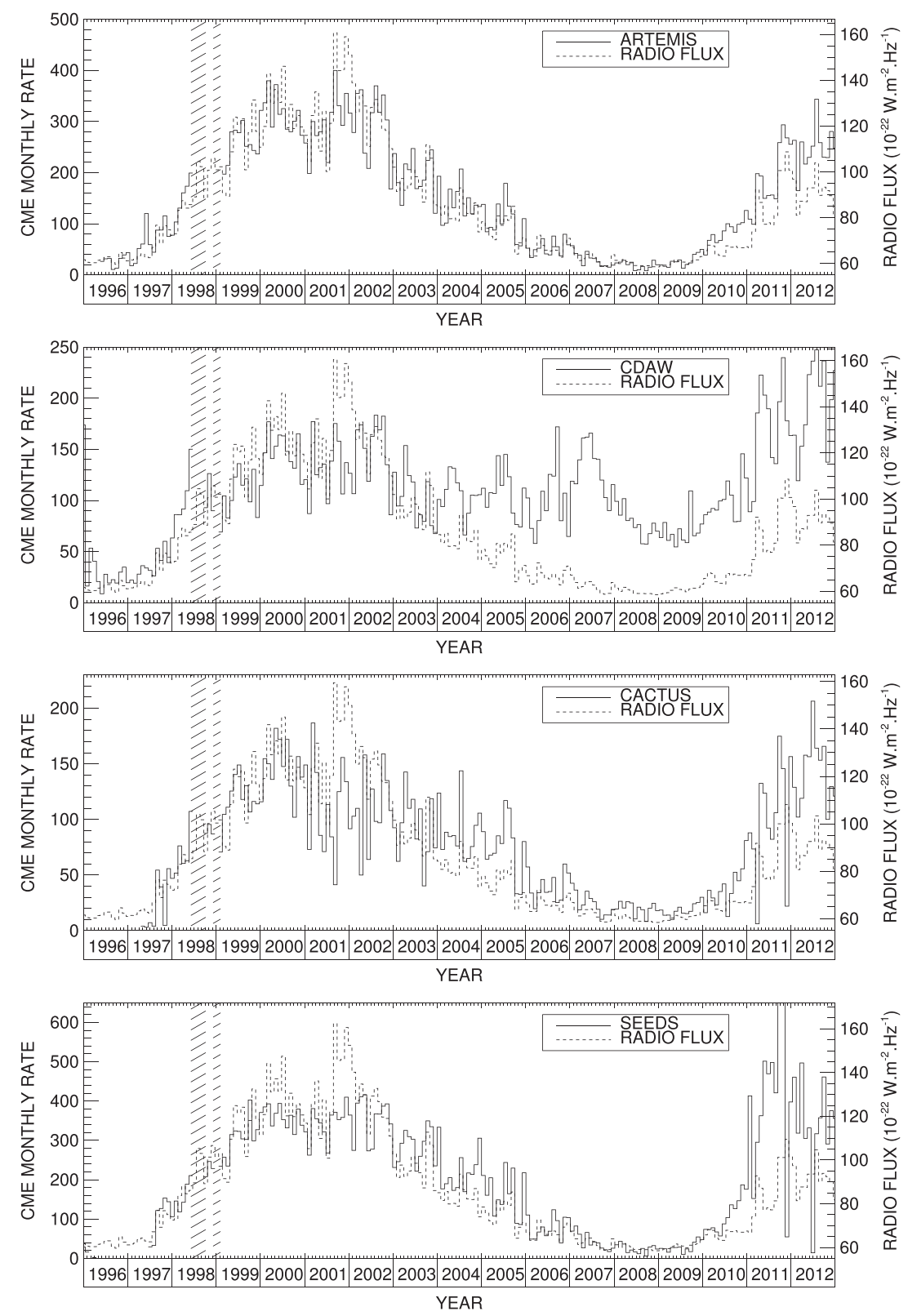

Figure 8. Monthly rates of coronal mass ejections reported by the ARTEMIS-II, CACTUS, CDAW, and SEEDS catalogs and the $F_{10.7}$ radio flux. Note the different vertical scales.

\section{Temporal Variations of the Rate of Coronal Mass Ejections}

We finally consider the relationship between solar activity and coronal mass ejections (CME). Figure 8 displays their monthly rate of occurrence derived from the ARTEMIS-II catalog together with the $F_{10.7}$ index; note the excellent agreement, even at the level of many temporal substructures. This figure further displays the rates given by three other catalogs of CME, the Coordinated Data Analysis Workshop (CDAW, http://cdaw.gsfc.nasa.gov/CME_list/) based on visual detection [Yashiro et al., 2004], the Computer Aided CME Tracking catalog (CACTus, http://sidc.oma.be/cactus/) based on automatic detection [Robbrecht and Berghmans, 2004], and the Solar Eruptive Event Detection System (SEEDS, http://spaceweather.gmu.edu/ seeds/) also based on automatic detection [Olmedo et al., 2008].

Altogether, the monthly rates given by the three automated catalogs follow quite well the pattern of solar activity (with some second-order differences) and, in particular, reach very low levels during the cycle 23/24 
minimum. This is not the case of the rate given by CDAW which diverges from the index in 2004 during the declining phase of solar cycle 23 and thereafter remains at a rather high level during the following minimum, except for about a year. We stress that the CDAW catalog depends on the human selection factor and therefore does not have the same uniform calibration as the others. Only CDAW and ARTEMIS cover the first 2 years of LASCO operation (1996 and 1997). The ARTEMIS catalog indicates a pronounced cycle 23/24 minimum extending from September 2007 to October 2009 with a mean rate apparently slightly lower (by a factor of roughly 0.7 ) than the mean rate observed in 1996 and early 1997 . The CDAW catalog indicates a minimum lasting approximately 1 year overlapping 2008 and 2009 but at a much higher rate than the minimum observed in 1996/1997 (typically a factor of 3 larger) which is difficult to understand in view of the consistency between the three automated catalogs. As a final positive note, let us mention that all four catalogs agree on the fact that the CME rate has been increasing faster than the activity index (SSN and $F_{10.7}$ ) during the rising phase of solar cycle 24 as already pointed out by Luhmann et al. [2011].

\section{Discussion}

The temporal variation of the global radiance of the corona (Figure 3) suggests that it reached an absolute low plateau during an interval of approximately 1.5 years roughly centered on January 2009 , a length apparently comparable to the plateau of cycle 22/23 minimum if we assume that the LASCO observations did not truncate that minimum (this is plausible but not fully ascertained as LASCO did not record the descending branch of the cycle). However, it is worth pointing out that the time during which the global radiance of the cycle $23 / 24$ minimum was below that of cycle $22 / 23$ amounts to $\approx 3$ years and even $\approx 3.5$ years if we neglect the slight bump of early 2010. Let us now examine this question in different sectors. In the two hemispheres, the low plateaus lasted about the same duration, possibly slightly longer, approximately 1.7 year. The situation is entirely different in the equatorial sector where the radiance persisted at a constant low level for about 4 years. It is contrasted in the polar sectors with durations of $\approx 1.7$ year at the north and $\approx 2.2$ year at the south (Figure 4). Another view is offered by inspecting the angular width (i.e., latitudinal extent) of the streamer belt and estimating the time during which it was minimal (Figure 7). Using this criterion, we can determine a length of $\approx 0.3$ year for the cycle $22 / 23$ minimum and a much longer length of $\approx 1$ year for that of cycle 23/24 with a broader extent as noted in section 4 .

The different durations of the minimum phases of the global radiance dot not allow us to define what could be considered the length of cycle 23. The time shift of the ascending branches as calculated in section 3 represents the best estimate that we can derive from coronal observations, that is 12 years and 3 months. To have a meaningful comparison with solar activity index, we performed the same exercise of correlating the ascending branches of the temporal variations of the sunspot number and of the $F_{10.7} \mathrm{~cm}$ radio flux (using 13 months smoothing) and obtained respectively 12 years and 6 months and 12 years and 5 months. We do not consider these differences of respectively 3 and 2 months to be significant but only point out that these values are in agreement on being substantially larger that the classical duration of a solar cycle of 11 years. Yet these values are not exceptional and remain well below the maximum of $\approx 13.5$ years recorded in history [see Hathaway, 2010, Figure 22]. According to the amplitude-period effect, the maximum sunspot number of cycle 24 should not exceed 100 [see Hathaway, 2010, Figure 27] well in line with the present observations.

As far as the radiances of the global corona and of that of the two hemispheres are concerned, the onsets of activity (as determined from the ascending branches) of cycles 23 and 24 look quite similar. This is not the case of the polar sectors. In the north one, the two onsets are in phase assuming a time shift of 147 months, but at the south, the onset of cycle 24 is delayed by $\approx 7$ months.

Our results are well in line with the general characterization of the anomalous minimum of solar cycle $23 / 24$ based on a variety of studies. The temporal variation of the global coronal radiance, and particularly during this minimum, closely follows the pattern of the standard proxies of solar activity, SSN and $F_{10.7}$. This is also the case of other proxies (TSI, Mg II, Ca II, UV, and EUV irradiances) although the ratios between the cycles 22/23 and 23/24 minima differ, probably reflecting different physical conditions and processes at work. Directly related to our results in the equatorial region are the in situ measurements of the proton density at $1 \mathrm{AU}$ in the ecliptic plane obtained by Wind and ACE. The yearly averages during the minima of cycles $22 / 23$ and $23 / 24$ of respectively 8.3 and $4.4 \mathrm{~cm}^{-3}$ [see Wu et al., 2013, Figure 1] lead to a 
ratio of 0.53 in astonishing agreement with our value of 0.56 . The global 3-D, time-dependent, numerical MHD model of Wu et al. [2013] was able to reproduce the in situ measurements and further showed that the polar regions were much less affected than the equatorial region, here again in agreement with our results.

The observed latitudinal spread of the streamer belt during the cycle 23/24 minimum is consistent with the fact that the tilt angle of the heliocentric current sheet remained anomalously large during several years as illustrated by Manoharan [2012, Figure 7]. This figure, as well as Petrie's [2013, Figures 1 and 3], further shows that the polar field remained rather low during this period (see also Wang et al. [2009]) with the consequences that the low-latitude region plasma was less compressed and that the large-scale magnetic field of the Sun did not approach the classical dipole geometry as seen on the LASCO images of the corona and the derived synoptic maps. Indeed, the solar wind global pressure as measured in situ in the ecliptic plane was the lowest observed since the beginning of the space age [Luhmann et al., 2009].

The asymmetry between the north and south hemispheres has been noted in numerous works [e.g., Petrie, 2012; Gopalswamy et al., 2012; McIntosh, 2013] and is intimately connected to the phase lag of the onset of cycle 24 activity between the two polar regions. According to observations of the "rush to the poles" of the polar crown prominences and their associated coronal Fe XIV emission features [Altrock, 2013], of prominence eruption activity in microwave brightness observations [Gopalswamy et al., 2012], and to the marked difference between the north and south sunspot areas, solar maximum conditions have been reached in the northern region, approximately in late 2012, whereas the southern region is lagging with the rise of activity still ongoing. This picture is very well reflected in our results, not so much in the radiance calculated in the two hemispheres (because of the dominant contribution of the equatorial belt) but conspicuously in that calculated in the two polar regions (Figure 5). This hemispheric phase difference appears to belong to a broad secular variation with reversals occurring roughly every eight solar cycles; the last one took place in 1968 and saw the Northern Hemisphere taking over the Southern one [Zolotova et al., 2010].

\section{Conclusion}

Not only can the solar cycle be traced high into the corona [Tappin and Altrock, 2013], the analysis of properly processed and calibrated white light images of the LASCO-C2 coronagraph yields both quantitative results (radiance, polarized radiance, and electron density) and spatially resolved information in regions inaccessible to in situ measurements (e.g., polar regions) giving a unique and broad insight on how the corona reacts to the solar activity. Our main conclusions from this study mainly devoted to comparing the minima of solar cycles $22 / 23$ and $23 / 24$ are summarized below.

1. The temporal variation of the global radiance and henceforth of the total electron content of the $K$ corona follow very well the standard proxies of solar activity, SSN and $F_{10.7}$.

2. The global radiance of the $K$ corona in the annular region extending from 2.7 to $5.5 \mathrm{R}_{\odot}$ was $24 \%$ fainter during the cycle $23 / 24$ minimum than during the previous minimum.

3. This global result hides a more complex situation as the two hemispheres experienced different reductions, $17 \%$ for the north one and $29 \%$ for the south one.

4. The differences are even more pronounced when considering restricted angular sectors $30^{\circ}$ wide: The equatorial sector suffered a drastic reduction of $44 \%$, whereas the north and south polar sectors did not experience much variation.

5. This reduction in the equatorial sector is in remarkable agreement with the in situ measurements of the proton density performed at $1 \mathrm{AU}$ in the ecliptic plane by Wind and ACE.

6. The phasing of the ascending branches of the $K$ corona radiance of cycles 23 and 24 yields an estimate of 12 years and 3 months for the duration of cycle 23.

7. The asymmetry between the north and south hemispheres and specifically the north and south sectors is well observed in the LASCO-C2 data. Our results support the current view that solar maximum conditions have been reached in the northern region, whereas the southern region is lagging with the rise of activity still ongoing.

8. The three catalogs of coronal mass ejections based on automatic detection agree in showing that their rate follows well the activity pattern of the Sun with pronounced minima at the end of solar cycles 22 and 23. 


\section{Acknowledgments}

The LASCO-C2 project at the Laboratoire d'Astrophysique de Marseille is funded by the Centre National d'Etudes Spatiales (CNES). LASCO was built by a consortium of the Naval Research Laboratory, USA, the Laboratoire d'Astrophysique de Marseille (formerly Laboratoire d'Astronomie Spatiale), France, the Max-Planck-Institut für Sonnensystemforschung (formerly Max Planck Institute für Aeronomie), Germany, and the School of Physics and Astronomy, University of Birmingham, UK. SOHO is a project of international cooperation between ESA and NASA.

Philippa Browning thanks the reviewers for their assistance in evaluating this paper.
We will continue monitoring the activity of the corona as cycle 24 develops and hopefully to the next minimum if $\mathrm{SOHO}$ continues its incredible saga. As already mentioned in the text, our next and already ongoing effort concerns the analysis and interpretation of the temporal evolution of the corona over a cycle and a half (Barlyaeva et al., manuscript in preparation).

\section{References}

Allen, C. W. (1973), Astrophysical Quantities, 3rd ed., The Athlon Press, University of London, London, U. K.

Altrock, R. C. (2013), Forecasting the maxima of solar cycle 24 with coronal Fe XIV emission, Sol. Phys., 289, 623-629.

Boursier, Y., P. Lamy, A. Llebaria, F. Goudail, and S. Robelus (2009), The ARTEMIS catalog of LASCO coronal mass ejections, Sol. Phys., 257, 125-147.

Brueckner, G. E., et al. (1995), The large angle spectroscopic coronagraph (LASCO), Sol. Phys., 162, 357-402.

Fisher, R., and D. G. Sime (1984), Solar activity cycle variation of the K corona, Astrophys. J., 285, 354-358.

Floyd, O., P. Lamy, Y. Boursier, and A. Llebaria (2013), ARTEMIS II: A second-generation catalog of LASCO coronal mass ejections including mass and kinetic energy, Sol. Phys., 288(1), 269-289.

Gardès, B., P. Lamy, and A. Llebaria (2013), Photometric calibration of the LASCO-C2 coronagraph over 14 years (1996-2009), Sol. Phys., 283(2), 667-690.

Gopalswamy, N., S. Yashiro, P. Mäkelä, G. Michalek, K. Shibasaki, and D. H. Hathaway (2012), Erratum: “Behavior of solar cycles 23 and 24 revealed by microwave observations", Astrophys. J., 750(2), L42.

Hathaway, D. H. (2010), The Solar Cycle, Living Rev. Sol. Phys., 7, 1-59.

Lamy, P., E. Quemerais, A. Llebaria, M. Bout, R. Howard, and R. Schwenn (1997), Electron densities in coronal holes from LASCO-C2 images, in Fifth SOHO Workshop: The Corona and Solar Wind Near Minimum Activity. held at Institute of Theoretical Astrophysics. University of Oslo, Norway, 17-20 June, 1997, vol. 404, edited by A. Wilson, pp. 491-494, European Space Agency, Noordwijk, Netherlands.

Lamy, P., A. Llebaria, and E. Quémerais (2002), Solar cycle variation of the radiance and the global electron density of the solar corona, Adv. Space Res., 29(3), 373-378.

Llebaria, A., P. Lamy, and S. Koutchmy (1999), The global activity of the solar corona, in 8th SOHO Workshop: Plasma Dynamics and Diagnostics in the Solar Transition Region and Corona. Proceedings of the Conference held 22-25 June 1999 in CAP 15, 1-13 Quai de Grenelle, 75015 Paris, France, Sponsored by ESA, NASA, C.N.R.S.-I.N.S.U., Euroconferences, Institut d'Astrophysique Spatiale, Matra Marconi Space, SCOSTEP, vol. 466, edited by J.-C. Vial and B. Kaldeich-Schümann, pp. 441-446, ESA Special Publications, Université Paris XI, Paris, France.

Llebaria, A., and A. F. R. Thernisien (2001), Highly accurate photometric equalization of long sequences of coronal images, in Proceedings of SPIE, Astronomical Data Analysis Workshop, vol. 4477, edited by J.-L. Starck and F. D. Murtagh, pp. 265-276, SPIE, Bellingham, Wash

Llebaria, A., P. Lamy, and M. V. Bout (2004), Lessons learned from the SOHO/LASCO-C2 calibration, in Proceedings of the SPIE, Telescopes and Instrumentation for Solar Astrophysics, vol. 5171, edited by S. Fineschi and M. A. Gummin, pp. 26-37, SPIE, Bellingham, Wash.

Llebaria, A., P. Lamy, and J.-F. Danjard (2006), Photometric calibration of the LASCO-C2 coronagraph for solar system objects, Icarus, 182, 281-296.

Llebaria, A., and P. Lamy (2008), In-orbit calibration of the polarization flat fields of the SOHO-LASCO coronagraphs, in Proceedings-SPIE The International Society For Optical Engineering, Space Telescopes and Instrumentation: Optical, Infrared and Millimeter; Space Telescopes and Instrumentation 2008, 7010, edited by J. M. Oschmann Jr., M. W. M. de Graauw, and H. A. MacEwen, pp. 12, SPIE, Bellingham, Wash.

Luhmann, J. G., C. O. Lee, Y. Li, C. N. Arge, A. B. Galvin, K. Simunac, C. T. Russell, R. A. Howard, and G. Petrie (2009), Solar wind sources in the late declining phase of cycle 23: Effects of the weak solar polar field on high speed streams, Sol. Phys., 256, $285-305$.

Luhmann, J. G., Y. Li, Y. Liu, L. Jian, C. T. Russell, E. Kilpua, G. J. Petrie, and J. T. Hoeksema (2011), Heliospheric space weather at the start of cycle 24, Abstract SH31D-04 presented at 2011 Fall Meeting, AGU, San Francisco, Calif., 5-9 Dec.

Manoharan, P. K. (2012), Three-dimentional evolution of solar wind during solar cycles 22-24, 751, 128, 13.

McIntosh, S. W., R. J. Leamon, J. B. Gurman, J.-Ph. Olive, J. W. Cirtain, D. H. Hathaway, J. Burkepile, M. Miesch, R. S. Markel, and L. Sitongia (2013), Hemispheric assymmetries of solar photospheric magnetism: Radiative, particulate, and heliospheric impacts, Astrophys. J., $765,146,17$.

Olmedo, O., J. Zhang, H. Wechsler, A. Poland, and K. Borne (2008), Automatic detection and tracking of coronal mass ejections in coronagraph time series, Sol. Phys., 248, 485-499.

Pagot, E., P. Lamy, A. Llebaria, and B. Boclet (2013), Automated processing of LASCO coronal images: Spurious point-source filtering and missing blocks correction, Sol. Phys., 289, 1433-1453.

Petrie, G. J. D. (2012), Evolution of active and polar photospheric magnetic fields during the rise of cycle 24 compared to previous cycles, Sol. Phys., 281, 577-598.

Petrie, G. J. D. (2013), Solar magnetic activity cycles, coronal potential field models and eruption rates, Astrophys. J., 768, 162,18.

Robbrecht, E., and D. Berghmans (2004), Automated recognition of coronal mass ejections (CMEs) in near-real-time data, Astron. Astrophys., 425, 1097-1106.

Tappin, S. J., and R. C. Altrock (2013), The extended solar cycle tracked high into the corona, Sol. Phys., 282(1), $249-261$.

van de Hulst, H. C. (1950), The electron density of the solar corona, Bull. Astron. Inst. Neth., 11, 135-150.

Wang, Y. M., E. Robbrecht, and N. R. Sheeley Jr (2009), On the weakening of the polar magnetic fields during solar cycle 23, Astrophys. J., 707, 1372-1386.

Wu, C.-C., K. Liou, S. Plunkett, C. D. Fry, and S.-T. Wu (2013), Investigation of solar/heliospheric anomalies associated with the solar minimum of 2007-2008, Terr. Atmos. Ocean. Sci., 24(2), 243-252.

Yashiro, S., N. Gopalswamy, G. Michalek, St. O. C. Cyr, S. Plunkett, N. Rich, and R. Howard (2004), A catalog of white light coronal mass ejections observed by the SOHO spacecraft, J. Geophys. Res., 109, A07105, doi:10.1029/2003JA010282.

Zolotova, N. V., D. I. Ponyavin, R. Arlt, and I. Tuominen (2010), Secular variation of hemispheric phase differences in the solar cycle, Astron. Nachr., 331, 765-771. 